\title{
DIFFERENTIAL POLYNOMIALS GENERATED BY SECOND ORDER LINEAR DIFFERENTIAL EQUATIONS
}

\author{
B. BELAÏDI and A. EL FARISSI
}

Received December 5, 2007 and, in revised form, July 14, 2008

\begin{abstract}
In this paper, we study fixed points of solutions of the differential equation

$$
f^{\prime \prime}+A_{1}(z) f^{\prime}+A_{0}(z) f=0
$$

where $A_{j}(z)(\not \equiv 0)(j=0,1)$ are transcendental meromorphic functions with finite order. Instead of looking at the zeros of $f(z)-z$, we proceed to a slight generalization by considering zeros of $g(z)-\varphi(z)$, where $g$ is a differential polynomial in $f$ with polynomial coefficients, $\varphi$ is a small meromorphic function relative to $f$, while the solution $f$ is of infinite order.
\end{abstract}

\section{Introduction AND MAIN RESUlts}

Throughout this paper, we assume that the reader is familiar with the fundamental results and the standard notations of the Nevanlinna's value distribution theory and with the basic Wiman Valiron theory as well (see

2000 Mathematics Subject Classification. Primary: 34M10, 30D35.

Key words and phrases. Linear differential equations, meromorphic solutions, hyper order, exponent of convergence of the sequence of distinct zeros, hyper exponent of convergence of the sequence of distinct zeros.

ISSN 1425-6908（C) Heldermann Verlag. 
[7], [8], [10], [13], [14]). In addition, we will use $\lambda(f)$ and $\lambda(1 / f)$ to denote respectively the exponents of convergence of the zero-sequence and the polesequence of a meromorphic function $f, \rho(f)$ to denote the order of growth of $f, \bar{\lambda}(f)$ and $\bar{\lambda}(1 / f)$ to denote respectively the exponents of convergence of the sequence of distinct zeros and distinct poles of $f$. A meromorphic function $\varphi(z)$ is called a small function of a meromorphic function $f(z)$ if $T(r, \varphi)=o(T(r, f))$ as $r \rightarrow+\infty$, where $T(r, f)$ is the Nevanlinna characteristic function of $f$. In order to express the rate of growth of meromorphic solutions of infinite order, we recall the following definition.

Definition 1.1 ([2], [12], [16]). Let $f$ be a meromorphic function. Then the hyper order $\rho_{2}(f)$ of $f(z)$ is defined by

$$
\rho_{2}(f)=\varlimsup_{r \rightarrow+\infty} \frac{\log \log T(r, f)}{\log r} .
$$

Definition $1.2([2],[9],[12])$. Let $f$ be a meromorphic function. Then the hyper exponent of convergence of the sequence of distinct zeros of $f(z)$ is defined by

$$
\bar{\lambda}_{2}(f)=\varlimsup_{r \rightarrow+\infty} \frac{\log \log \bar{N}\left(r, \frac{1}{f}\right)}{\log r},
$$

where $\bar{N}(r, 1 / f)$ is the counting function of distinct zeros of $f(z)$ in $\{|z|<r\}$.

Consider the second order linear differential equation

$$
f^{\prime \prime}+A_{1}(z) f^{\prime}+A_{0}(z) f=0,
$$

where $A_{j}(z)(\not \equiv 0)(j=0,1)$ are transcendental meromorphic functions with finite order. Many important results have been obtained on the fixed points of general transcendental meromorphic functions for almost four decades (see [17]). However, there are a few studies on the fixed points of solutions of differential equations. It was in year 2000 that Z. X. Chen first pointed out the relation between the exponent of convergence of distinct fixed points and the rate of growth of solutions of second order linear differential equations with entire coefficients (see [2]). In [15], Wang and Yi investigated fixed points and hyper order of differential polynomials generated by solutions of second order linear differential equations with meromorphic coefficients. In [11], Laine and Rieppo gave improvement of the results of [15] by considering fixed points and iterated order. In [3], Chen Zongxuan and Shon Kwang Ho have studied the differential equation

$$
f^{\prime \prime}+A_{1}(z) e^{a z} f^{\prime}+A_{0}(z) e^{b z} f=0
$$


and have obtained the following results:

Theorem A $([3])$. Let $A_{j}(z)(\not \equiv 0)(j=0,1)$ be meromorphic functions with $\rho\left(A_{j}\right)<1(j=0,1), a, b$ be complex numbers such that $a b \neq 0$ and $\arg a \neq \arg b$ or $a=c b(0<c<1)$. Then every meromorphic solution $f(z) \not \equiv 0$ of the equation (1.4) has infinite order.

In the same paper, Z. X. Chen and K. H. Shon have investigated the fixed points of solutions, their $1^{\text {st }}, 2^{\text {nd }}$ derivatives and differential polynomial and have obtained:

Theorem B $([3])$. Let $A_{j}(z)(j=0,1), a, b, c$ satisfy the additional hypotheses of Theorem $A$. Let $d_{0}, d_{1}, d_{2}$ be complex constants that are not all equal to zero. If $f(z) \not \equiv 0$ is any meromorphic solution of equation (1.4), then:

(i) $f, f^{\prime}, f^{\prime \prime}$ all have infinitely many fixed points and satisfy

$$
\bar{\lambda}(f-z)=\bar{\lambda}\left(f^{\prime}-z\right)=\bar{\lambda}\left(f^{\prime \prime}-z\right)=+\infty,
$$

(ii) the differential polynomial

$$
g(z)=d_{2} f^{\prime \prime}+d_{1} f^{\prime}+d_{0} f
$$

has infinitely many fixed points and satisfies $\bar{\lambda}(g-z)=+\infty$.

In this paper, we study the relation between the small functions and solutions of equation (1.3) in the case when all meromorphic solutions are of infinite order and we obtain the following results:

Theorem 1.1. Let $A_{j}(z)(\not \equiv 0)(j=0,1)$ be transcendental meromorphic functions with finite order such that all meromorphic solutions of equation (1.3) are of infinite order, let $d_{j}(j=0,1,2)$ be polynomials that are not all equal to zero, $\varphi(z)(\not \equiv 0)$ be a meromorphic function of finite order satisfying

$$
\left(d_{1}-d_{2} A_{1}\right) \varphi^{\prime}-\left(d_{2} A_{1}^{2}-\left(d_{2} A_{1}\right)^{\prime}-d_{2} A_{0}-d_{1} A_{1}+d_{0}+d_{1}^{\prime}\right) \varphi \not \equiv 0 .
$$

If $f \not \equiv 0$ is a meromorphic solution of equation (1.3) with $\lambda(1 / f)<$ $+\infty$, then the differential polynomial $g(z)=d_{2} f^{\prime \prime}+d_{1} f^{\prime}+d_{0} f$ satisfies $\bar{\lambda}(g-\varphi)=+\infty$.

Theorem 1.2. Suppose that $A_{j}(z)(\not \equiv 0)(j=0,1), \varphi(z) \not \equiv 0$ satisfy the hypotheses of Theorem 1.1. If $f(z) \not \equiv 0$ is a meromorphic solution of (1.3) with $\rho(f)=+\infty$ and $\rho_{2}(f)=\rho$, then $f$ satisfies

$$
\begin{aligned}
& \bar{\lambda}(f-\varphi)=\bar{\lambda}\left(f^{\prime}-\varphi\right)=\bar{\lambda}\left(f^{\prime \prime}-\varphi\right)=\rho(f)=+\infty, \\
& \bar{\lambda}_{2}(f-\varphi)=\bar{\lambda}_{2}\left(f^{\prime}-\varphi\right)=\bar{\lambda}_{2}\left(f^{\prime \prime}-\varphi\right)=\rho_{2}(f)=\rho .
\end{aligned}
$$


Corollary. Let $A_{0}(z)$ be a transcendental entire function with $\rho\left(A_{0}\right)<1$, let $d_{j}(j=0,1,2)$ be polynomials that are not all equal to zero, $\varphi(z)(\not \equiv 0)$ be an entire function of finite order. If $f$ is a nontrivial solution of the equation

$$
f^{\prime \prime}+e^{-z} f^{\prime}+A_{0}(z) f=0,
$$

then the differential polynomial $g(z)=d_{2} f^{\prime \prime}+d_{1} f^{\prime}+d_{0} f$ satisfies $\bar{\lambda}(g-\varphi)=+\infty$.

\section{Auxiliary lemmas}

We need the following lemmas in the proofs of our theorems.

Lemma $2.1([6])$. Let $f$ be a transcendental meromorphic function of finite order $\rho$, let $\Gamma=\left\{\left(k_{1}, j_{1}\right),\left(k_{2}, j_{2}\right), \ldots,\left(k_{m}, j_{m}\right)\right\}$ denote a finite set of distinct pairs of integers that satisfy $k_{i}>j_{i} \geq 0$ for $i=1, \ldots, m$ and let $\varepsilon>0$ be a given constant. Then the following two statements hold:

(i) There exists a set $E_{1} \subset[0,2 \pi)$ that has linear measure zero, such that if $\psi \in[0,2 \pi) \backslash E_{1}$, then there is a constant $R_{1}=R_{1}(\psi)>1$ such that for all $z$ satisfying $\arg z=\psi$ and $|z| \geq R_{1}$ and for all $(k, j) \in \Gamma$, we have

$$
\left|\frac{f^{(k)}(z)}{f^{(j)}(z)}\right| \leq|z|^{(k-j)(\rho-1+\varepsilon)} .
$$

(ii) There exists a set $E_{2} \subset(1,+\infty)$ that has finite logarithmic measure

$$
\operatorname{lm}\left(E_{2}\right)=\int_{1}^{+\infty} \frac{\chi_{E_{2}}(t)}{t} d t
$$

where $\chi_{E_{2}}$ is the characteristic function of $E_{2}$, such that for all $z$ satisfying $|z| \notin E_{2} \cup[0,1]$ and for all $(k, j) \in \Gamma$, we have

$$
\left|\frac{f^{(k)}(z)}{f^{(j)}(z)}\right| \leq|z|^{(k-j)(\rho-1+\varepsilon)} .
$$

Lemma 2.2. Let $A_{j}(j=0,1)$ and $A_{2}(\not \equiv 0)$ be meromorphic functions with $\rho\left(A_{j}\right)<1(j=0,1,2)$. We denote $\psi_{2}(z)=A_{0}+A_{1} e^{-z}$. Let $\psi_{21}$, $\psi_{20}$ have the form of $\psi_{2}$, and $\varphi \not \equiv 0$ be a meromorphic function of finite order. Then

(i) $\psi_{2}(z)+A_{2} e^{-2 z} \not \equiv 0$;

(ii) $\psi_{21} \frac{\varphi^{\prime}}{\varphi}+\psi_{20}+A_{2} e^{-2 z} \not \equiv 0$. 
Proof. Suppose that the claim fails. As for (i), if $A_{2} e^{-2 z}+A_{1} e^{-z}+A_{0} \equiv 0$, then

$$
\begin{aligned}
2 T\left(r, e^{-z}\right) & \leq T\left(r, A_{2} e^{-2 z}\right)+T\left(r, \frac{1}{A_{2}}\right) \\
& =T\left(r, A_{1} e^{-z}+A_{0}\right)+T\left(r, \frac{1}{A_{2}}\right) \\
& \leq T\left(r, e^{-z}\right)+\sum_{j=0}^{2} T\left(r, A_{j}\right)+O(1),
\end{aligned}
$$

hence $\rho\left(e^{-z}\right)<1$, a contradiction. As for (ii), the left hand side can be written as follows

$$
\begin{aligned}
& \psi_{21} \frac{\varphi^{\prime}}{\varphi}+\psi_{20}+A_{2} e^{-2 z} \\
& =A_{00}+A_{01} \frac{\varphi^{\prime}}{\varphi}+\left(A_{10}+A_{11} \frac{\varphi^{\prime}}{\varphi}\right) e^{-z}+A_{2} e^{-2 z}
\end{aligned}
$$

where $A_{00}, A_{01}, A_{10}, A_{11}$ are meromorphic functions of order $<1$. Since $\varphi$ is of finite order, then by the lemma of logarithmic derivative $([7])$

$$
m\left(r, \frac{\varphi^{\prime}}{\varphi}\right)=O(\ln r)
$$

Therefore, by a reasoning as to above, but using the proximity functions instead of the characteristic, a contradiction $\rho\left(e^{-z}\right)<1$ again follows.

Lemma $2.3([4])$. If $A_{0}(z)$ is a transcendental entire function with $\rho\left(A_{0}\right)<1$, then every solution $f(z) \not \equiv 0$ of equation (1.8) has infinite order.

Lemma 2.4 (Wiman-Valiron, [8], [14]). Let $f(z)$ be a transcendental entire function, and let $z$ be a point with $|z|=r$ at which $|f(z)|=M(r, f)$. Then the estimate

$$
\frac{f^{(k)}(z)}{f(z)}=\left(\frac{\nu(r, f)}{z}\right)^{k}(1+o(1)) \quad(k \geq 1 \text { is an integer })
$$

holds for all $|z|$ outside a set $E_{3}$ of $r$ of finite logarithmic measure, where $\nu(r, f)$ denotes the central index of $f$.

To avoid some problems caused by the exceptional set we recall the following lemma. 
Lemma $2.5([5])$. Let $g:[0,+\infty) \rightarrow \mathbb{R}$ and $h:[0,+\infty) \rightarrow \mathbb{R}$ be monotone non-decreasing functions such that $g(r) \leq h(r)$ for all $r \notin E_{4} \cup[0,1]$, where $E_{4} \subset(1,+\infty)$ is a set of finite logarithmic measure. Let $\alpha>1$ be a given constant. Then there exists an $r_{0}=r_{0}(\alpha)>0$ such that $g(r) \leq h(\alpha r)$ for all $r \geq r_{0}$.

Lemma 2.6. Let $f(z)$ be a meromorphic function with $\rho(f)=+\infty$ and the exponent of convergence of the poles of $f(z), \lambda(1 / f)<+\infty$. Let $d_{j}(z)$ $(j=0,1,2)$ be polynomials that are not all equal to zero. Then

$$
g(z)=d_{2}(z) f^{\prime \prime}+d_{1}(z) f^{\prime}+d_{0}(z) f
$$

satisfies $\rho(g)=+\infty$.

Proof. We suppose that $\rho(g)<+\infty$ and then we obtain a contradiction. First, we suppose that $d_{2}(z) \not \equiv 0$. Set $f(z)=w(z) / h(z)$, where $h(z)$ is canonical product (or polynomial) formed with the non-zero poles of $f(z), \lambda(h)=\rho(h)=\lambda(1 / f)<+\infty, w(z)$ is an entire function with $\rho(w)=\rho(f)=+\infty$. We have

$$
\begin{aligned}
f^{\prime}(z) & =\frac{w^{\prime}}{h}-\frac{h^{\prime}}{h^{2}} w \\
f^{\prime \prime}(z) & =\frac{w^{\prime \prime}}{h}-2 \frac{h^{\prime}}{h^{2}} w^{\prime}+\left(2 \frac{\left(h^{\prime}\right)^{2}}{h^{3}}-\frac{h^{\prime \prime}}{h^{2}}\right) w
\end{aligned}
$$

and

$$
\begin{aligned}
f^{\prime \prime \prime}(z)= & \frac{w^{\prime \prime \prime}}{h}-3 \frac{h^{\prime}}{h^{2}} w^{\prime \prime}+\left(6 \frac{\left(h^{\prime}\right)^{2}}{h^{3}}-3 \frac{h^{\prime \prime}}{h^{2}}\right) w^{\prime} \\
& +\left(6 \frac{h^{\prime} h^{\prime \prime}}{h^{3}}-6 \frac{\left(h^{\prime}\right)^{3}}{h^{4}}-\frac{h^{\prime \prime \prime}}{h^{2}}\right) w .
\end{aligned}
$$

Hence

$$
\begin{aligned}
\frac{f^{\prime \prime \prime}(z)}{f(z)}= & \frac{w^{\prime \prime \prime}}{w}-3 \frac{h^{\prime}}{h} \frac{w^{\prime \prime}}{w}+\left(6 \frac{\left(h^{\prime}\right)^{2}}{h^{2}}-3 \frac{h^{\prime \prime}}{h}\right) \frac{w^{\prime}}{w} \\
& +6 \frac{h^{\prime} h^{\prime \prime}}{h^{2}}-6 \frac{\left(h^{\prime}\right)^{3}}{h^{3}}-\frac{h^{\prime \prime \prime}}{h}, \\
\frac{f^{\prime \prime}(z)}{f(z)}= & \frac{w^{\prime \prime}}{w}-2 \frac{h^{\prime}}{h} \frac{w^{\prime}}{w}+2 \frac{\left(h^{\prime}\right)^{2}}{h^{2}}-\frac{h^{\prime \prime}}{h}, \\
\frac{f^{\prime}(z)}{f(z)}= & \frac{w^{\prime}}{w}-\frac{h^{\prime}}{h} .
\end{aligned}
$$


Differentiating both sides of (2.7), we obtain

$$
g^{\prime}=d_{2} f^{\prime \prime \prime}+\left(d_{2}^{\prime}+d_{1}\right) f^{\prime \prime}+\left(d_{1}^{\prime}+d_{0}\right) f^{\prime}+d_{0}^{\prime} f .
$$

Writing $g^{\prime}=\left(g^{\prime} / g\right) g$ and substituting $g=d_{2} f^{\prime \prime}+d_{1} f^{\prime}+d_{0} f$ into (2.13), we get

$$
\begin{aligned}
& d_{2} f^{\prime \prime \prime}+\left(d_{2}^{\prime}-\frac{g^{\prime}}{g} d_{2}+d_{1}\right) f^{\prime \prime}+\left(d_{1}^{\prime}-\frac{g^{\prime}}{g} d_{1}+d_{0}\right) f^{\prime}+\left(d_{0}^{\prime}-\frac{g^{\prime}}{g} d_{0}\right) f \\
& =0
\end{aligned}
$$

This leads to

$$
\begin{aligned}
& d_{2} \frac{f^{\prime \prime \prime}}{f}+\left(d_{2}^{\prime}-\frac{g^{\prime}}{g} d_{2}+d_{1}\right) \frac{f^{\prime \prime}}{f}+\left(d_{1}^{\prime}-\frac{g^{\prime}}{g} d_{1}+d_{0}\right) \frac{f^{\prime}}{f}+\left(d_{0}^{\prime}-\frac{g^{\prime}}{g} d_{0}\right) \\
& =0 .
\end{aligned}
$$

Substituting (2.10)-(2.12) into (2.15), we obtain

$$
\begin{aligned}
& d_{2}\left(\frac{w^{\prime \prime \prime}}{w}-3 \frac{h^{\prime}}{h} \frac{w^{\prime \prime}}{w}+\left(6 \frac{\left(h^{\prime}\right)^{2}}{h^{2}}-3 \frac{h^{\prime \prime}}{h}\right) \frac{w^{\prime}}{w}+\left(6 \frac{h^{\prime} h^{\prime \prime}}{h^{2}}-6 \frac{\left(h^{\prime}\right)^{3}}{h^{3}}-\frac{h^{\prime \prime \prime}}{h}\right)\right) \\
& +\left(d_{2}^{\prime}-\frac{g^{\prime}}{g} d_{2}+d_{1}\right)\left(\frac{w^{\prime \prime}}{w}-2 \frac{h^{\prime}}{h} \frac{w^{\prime}}{w}+2 \frac{\left(h^{\prime}\right)^{2}}{h^{2}}-\frac{h^{\prime \prime}}{h}\right) \\
& +\left(d_{1}^{\prime}-\frac{g^{\prime}}{g} d_{1}+d_{0}\right)\left(\frac{w^{\prime}}{w}-\frac{h^{\prime}}{h}\right)+\left(d_{0}^{\prime}-\frac{g^{\prime}}{g} d_{0}\right)=0 .
\end{aligned}
$$

By Lemma 2.1 (ii), there exists a set $E_{1} \subset(1,+\infty)$ that has finite logarithmic measure, such that for all $z$ satisfying $|z|=r \notin E_{1} \cup[0,1]$, we have

$$
\left|\frac{g^{\prime}(z)}{g(z)}\right|=O\left(r^{\alpha}\right), \quad\left|\frac{h^{(j)}(z)}{h(z)}\right|=O\left(r^{\alpha}\right) \quad(j=1,2,3),
$$

where $0<\alpha<+\infty$ is some constant. By Lemma 2.4, there exists a set $E_{2} \subset(1,+\infty)$ with logarithmic measure $\operatorname{lm}\left(E_{2}\right)<+\infty$ and we can choose $z$ satisfying $|z|=r \notin E_{2} \cup[0,1]$ and $|w(z)|=M(r, w)$, such that

$$
\frac{w^{(j)}(z)}{w(z)}=\left(\frac{\nu(r, w)}{z}\right)^{j}(1+o(1)) \quad(j=1,2,3) .
$$

Now we take point $z$ satisfying $|z|=r \notin E_{1} \cup E_{2} \cup[0,1]$ and $w(z)=M(r, w)$, by substituting (2.17) and (2.18) into (2.16), we get

$$
\nu(r, w)=O\left(r^{\beta}\right),
$$

where $0<\beta<+\infty$ is some constant. By Lemma 2.5, we conclude that (2.19) holds for a sufficiently large $r$. This is a contradiction by $\rho(w)=+\infty$. Hence $\rho(g)=+\infty$. 
Now suppose $d_{2} \equiv 0, d_{1} \not \equiv 0$. Using a similar reasoning as above, we get a contradiction. Hence $\rho(g)=+\infty$.

Finally, if $d_{2} \equiv 0, d_{1} \equiv 0, d_{0} \not \equiv 0$, then we have $g(z)=d_{0}(z) f(z)$ and by $d_{0}$ is a polynomial, then we get $\rho(g)=+\infty$.

Lemma $2.7([1])$. Let $A_{0}, A_{1}, \ldots, A_{k-1}, F \not \equiv 0$ be finite order meromorphic functions. If $f$ is a meromorphic solution with $\rho(f)=+\infty$ of the equation

$$
f^{(k)}+A_{k-1} f^{(k-1)}+\cdots+A_{1} f^{\prime}+A_{0} f=F,
$$

then $\bar{\lambda}(f)=\lambda(f)=\rho(f)=+\infty$.

Lemma 2.8. Let $A_{0}, A_{1}, \ldots, A_{k-1}, F \not \equiv 0$ be finite order meromorphic functions. If $f$ is a meromorphic solution of the equation (2.20) with $\rho(f)=$ $+\infty$ and $\rho_{2}(f)=\rho$, then $f$ satisfies $\bar{\lambda}_{2}(f)=\lambda_{2}(f)=\rho_{2}(f)=\rho$.

Proof. By equation (2.20), we can write

$$
\frac{1}{f}=\frac{1}{F}\left(\frac{f^{(k)}}{f}+A_{k-1} \frac{f^{(k-1)}}{f}+\cdots+A_{1} \frac{f^{\prime}}{f}+A_{0}\right) .
$$

If $f$ has a zero at $z_{0}$ of order $\alpha(>k)$ and if $A_{0}, A_{1}, \ldots, A_{k-1}$ are all analytic at $z_{0}$, then $F$ has a zero at $z_{0}$ of order at least $\alpha-k$. Hence,

$$
n\left(r, \frac{1}{f}\right) \leq k \bar{n}\left(r, \frac{1}{f}\right)+n\left(r, \frac{1}{F}\right)+\sum_{j=0}^{k-1} n\left(r, A_{j}\right)
$$

and

$$
N\left(r, \frac{1}{f}\right) \leq k \bar{N}\left(r, \frac{1}{f}\right)+N\left(r, \frac{1}{F}\right)+\sum_{j=0}^{k-1} N\left(r, A_{j}\right) .
$$

By (2.21), we have

$$
m\left(r, \frac{1}{f}\right) \leq \sum_{j=1}^{k} m\left(r, \frac{f^{(j)}}{f}\right)+\sum_{j=0}^{k-1} m\left(r, A_{j}\right)+m\left(r, \frac{1}{F}\right)+O(1) .
$$

Applying the lemma of the logarithmic derivative (see [7]), we have

$$
m\left(r, \frac{f^{(j)}}{f}\right)=O(\log T(r, f)+\log r) \quad(j=1, \ldots, k),
$$

holds for all $r$ outside a set $E \subset(0,+\infty)$ with a finite linear measure $m(E)<$ $+\infty$. By (2.23), (2.24) and (2.25), we get

$$
T(r, f)=T\left(r, \frac{1}{f}\right)+O(1)
$$




$$
\begin{aligned}
\leq k \bar{N} & \left(r, \frac{1}{f}\right)+\sum_{j=0}^{k-1} T\left(r, A_{j}\right)+T(r, F)+O(\log (r T(r, f))) \\
& (|z|=r \notin E) .
\end{aligned}
$$

Since $\rho(f)=+\infty$, then there exists $\left\{r_{n}^{\prime}\right\}\left(r_{n}^{\prime} \rightarrow+\infty\right)$ such that

$$
\lim _{r_{n}^{\prime} \rightarrow+\infty} \frac{\log T\left(r_{n}^{\prime}, f\right)}{\log r_{n}^{\prime}}=+\infty .
$$

Set the linear measure of $E, m(E)=\delta<+\infty$, then there exists a point $r_{n} \in\left[r_{n}^{\prime}, r_{n}^{\prime}+\delta+1\right]-E$. From

$$
\begin{aligned}
\frac{\log T\left(r_{n}, f\right)}{\log r_{n}} & \geq \frac{\log T\left(r_{n}^{\prime}, f\right)}{\log \left(r_{n}^{\prime}+\delta+1\right)} \\
& =\frac{\log T\left(r_{n}^{\prime}, f\right)}{\log r_{n}^{\prime}+\log \left(1+\frac{\delta+1}{r_{n}^{\prime}}\right)}
\end{aligned}
$$

it follows that

$$
\lim _{r_{n} \rightarrow+\infty} \frac{\log T\left(r_{n}, f\right)}{\log r_{n}}=+\infty
$$

Set $\sigma=\max \left\{\rho\left(A_{j}\right)(j=0, \ldots, k-1), \rho(F)\right\}$. Then for a given arbitrary large $\beta>\sigma$,

$$
T\left(r_{n}, f\right) \geq r_{n}^{\beta}
$$

holds for sufficiently large $r_{n}$. On the other hand, for any given $\varepsilon$ with $0<2 \varepsilon<\beta-\sigma$, we have

$$
T\left(r_{n}, A_{j}\right) \leq r_{n}^{\sigma+\varepsilon}(j=0, \ldots, k-1), \quad T\left(r_{n}, F\right) \leq r_{n}^{\sigma+\varepsilon}
$$

for sufficiently large $r_{n}$. Hence, we have

$$
\max \left\{\frac{T\left(r_{n}, F\right)}{T\left(r_{n}, f\right)}, \frac{T\left(r_{n}, A_{j}\right)}{T\left(r_{n}, f\right)}(j=0, \ldots, k-1)\right\} \leq \frac{r_{n}^{\sigma+\varepsilon}}{r_{n}^{\beta}} \rightarrow 0,
$$

Therefore,

$$
\begin{aligned}
& T\left(r_{n}, F\right) \leq \frac{1}{k+3} T\left(r_{n}, f\right), \\
& T\left(r_{n}, A_{j}\right) \leq \frac{1}{k+3} T\left(r_{n}, f\right) \quad(j=0, \ldots, k-1)
\end{aligned}
$$

holds for sufficiently large $r_{n}$. From

$$
O\left(\log r_{n}+\log T\left(r_{n}, f\right)\right)=o\left(T\left(r_{n}, f\right)\right),
$$


we obtain that

$$
O\left(\log r_{n}+\log T\left(r_{n}, f\right)\right) \leq \frac{1}{k+3} T\left(r_{n}, f\right)
$$

also holds for sufficiently large $r_{n}$. Thus, by (2.26), (2.33), (2.35), we have

$$
T\left(r_{n}, f\right) \leq k(k+3) \bar{N}\left(r_{n}, \frac{1}{f}\right) .
$$

It yields $\bar{\lambda}_{2}(f)=\lambda_{2}(f)=\rho_{2}(f)=\rho$.

\section{Proof of Theorem 1.1}

First, we suppose $d_{2} \not \equiv 0$. Suppose that $f(\not \equiv 0)$ is a meromorphic solution of equation (1.3) with $\rho(f)=+\infty$ and $\lambda(1 / f)<+\infty$. Set $w=g-\varphi=$ $d_{2} f^{\prime \prime}+d_{1} f^{\prime}+d_{0} f-\varphi$, then by Lemma 2.6 we have $\rho(w)=\rho(g)=\rho(f)=$ $+\infty$. In order to the prove $\bar{\lambda}(g-\varphi)=+\infty$, we need to prove only $\bar{\lambda}(w)=$ $+\infty$.

Substituting $f^{\prime \prime}=-A_{1} f^{\prime}-A_{0} f$ into $w$, we get

$$
w=\left(d_{1}-d_{2} A_{1}\right) f^{\prime}+\left(d_{0}-d_{2} A_{0}\right) f-\varphi .
$$

Differentiating both a sides of equation (3.1), we obtain

$$
\begin{aligned}
w^{\prime}= & \left(d_{2} A_{1}^{2}-\left(d_{2} A_{1}\right)^{\prime}-d_{2} A_{0}-d_{1} A_{1}+d_{0}+d_{1}^{\prime}\right) f^{\prime} \\
& +\left(d_{2} A_{1} A_{0}-d_{1} A_{0}-\left(d_{2} A_{0}\right)^{\prime}+d_{0}^{\prime}\right) f-\varphi^{\prime} .
\end{aligned}
$$

Set

$$
\begin{aligned}
& \alpha_{1}=d_{1}-d_{2} A_{1}, \\
& \alpha_{0}=d_{0}-d_{2} A_{0}, \\
& \beta_{1}=d_{2} A_{1}^{2}-\left(d_{2} A_{1}\right)^{\prime}-d_{2} A_{0}-d_{1} A_{1}+d_{0}+d_{1}^{\prime}, \\
& \beta_{0}=d_{2} A_{1} A_{0}-d_{1} A_{0}-\left(d_{2} A_{0}\right)^{\prime}+d_{0}^{\prime} .
\end{aligned}
$$

Then we have

$$
\begin{aligned}
& \alpha_{1} f^{\prime}+\alpha_{0} f=w+\varphi, \\
& \beta_{1} f^{\prime}+\beta_{0} f=w^{\prime}+\varphi^{\prime} .
\end{aligned}
$$

Set

$$
h=\alpha_{1} \beta_{0}-\beta_{1} \alpha_{0} .
$$

We divide it into two cases to prove.

Case 1. If $h \equiv 0$, then by (3.3)-(3.5), we get

$$
\alpha_{1} w^{\prime}-\beta_{1} w=-\left(\alpha_{1} \varphi^{\prime}-\beta_{1} \varphi\right)=F .
$$


By the hypotheses of Theorem 1.1, we have $\alpha_{1} \varphi^{\prime}-\beta_{1} \varphi \not \equiv 0$ and then $F \not \equiv 0$. By $\alpha_{1} \not \equiv 0, F \not \equiv 0$, and Lemma 2.7, we obtain $\bar{\lambda}(w)=\lambda(w)=\rho(w)=+\infty$, i.e., $\bar{\lambda}(g-\varphi)=+\infty$.

Case 2. If $h \not \equiv 0$, then by (3.3)-(3.5), we get

$$
f=\frac{\alpha_{1}\left(w^{\prime}+\varphi^{\prime}\right)-\beta_{1}(w+\varphi)}{h} .
$$

Substituting (3.7) into equation (1.3) we obtain

$$
\begin{aligned}
& \frac{\alpha_{1}}{h} w^{\prime \prime \prime}+\phi_{2} w^{\prime \prime}+\phi_{1} w^{\prime}+\phi_{0} w \\
& =-\left(\left(\frac{\alpha_{1} \varphi^{\prime}-\beta_{1} \varphi}{h}\right)^{\prime \prime}+A_{1}\left(\frac{\alpha_{1} \varphi^{\prime}-\beta_{1} \varphi}{h}\right)^{\prime}+A_{0}\left(\frac{\alpha_{1} \varphi^{\prime}-\beta_{1} \varphi}{h}\right)\right) \\
& =F,
\end{aligned}
$$

where $\phi_{j}(j=0,1,2)$ are meromorphic functions with $\rho\left(\phi_{j}\right)<+\infty$ $(j=0,1,2)$. By the hypotheses of Theorem 1.1, we have $\alpha_{1} \varphi^{\prime}-\beta_{1} \varphi \not \equiv 0$ and by

$$
\rho\left(\frac{\alpha_{1} \varphi^{\prime}-\beta_{1} \varphi}{h}\right)<+\infty
$$

it follows that $F \not \equiv 0$. By Lemma 2.7, we obtain $\bar{\lambda}(w)=\lambda(w)=\rho(w)=$ $+\infty$, i.e., $\bar{\lambda}(g-\varphi)=+\infty$.

Now suppose $d_{2} \equiv 0, d_{1} \not \equiv 0$ or $d_{2} \equiv 0, d_{1} \equiv 0$ and $d_{0} \not \equiv 0$. Using a similar reasoning to that above we get $\bar{\lambda}(w)=\lambda(w)=\rho(w)=+\infty$, i.e., $\bar{\lambda}(g-\varphi)=+\infty$.

\section{Proof of Theorem 1.2}

Suppose that $f \not \equiv 0$ is a meromorphic solution of equation (1.3) with $\rho(f)=+\infty$ and $\rho_{2}(f)=\rho$. Set $w_{j}=f^{(j)}-\varphi(j=0,1,2)$. Since $\rho(\varphi)<$ $+\infty$, then we have $\rho\left(w_{j}\right)=\rho(f)=+\infty, \rho_{2}\left(w_{j}\right)=\rho_{2}(f)=\rho(j=0,1,2)$. By using a similar reasoning to that in the proof of Theorem 1.1, we obtain that

$$
\bar{\lambda}(f-\varphi)=\bar{\lambda}\left(f^{\prime}-\varphi\right)=\bar{\lambda}\left(f^{\prime \prime}-\varphi\right)=\rho(f)=+\infty
$$

and by Lemma 2.8, we get

$$
\bar{\lambda}_{2}(f-\varphi)=\bar{\lambda}_{2}\left(f^{\prime}-\varphi\right)=\bar{\lambda}_{2}\left(f^{\prime \prime}-\varphi\right)=\rho_{2}(f)=\rho .
$$




\section{Proof of Corollary}

Suppose that $f \not \equiv 0$ is a solution of equation (1.8). Then by Lemma 2.3, $f$ is of infinite order. Let $\varphi \not \equiv 0$ be a finite order entire function. Then by using Lemma 2.2, we have

$$
\begin{aligned}
& \left(d_{1}-d_{2} e^{-z}\right) \frac{\varphi^{\prime}}{\varphi} \\
& -\left(d_{2} e^{-2 z}-\left(d_{1}+d_{2}^{\prime}-d_{2}\right) e^{-z}-d_{2} A_{0}+d_{0}+d_{1}^{\prime}\right) \not \equiv 0 .
\end{aligned}
$$

Hence,

$$
\begin{aligned}
& \left(d_{1}-d_{2} e^{-z}\right) \varphi^{\prime} \\
& -\left(d_{2} e^{-2 z}-\left(d_{1}+d_{2}^{\prime}-d_{2}\right) e^{-z}-d_{2} A_{0}+d_{0}+d_{1}^{\prime}\right) \varphi \not \equiv 0 .
\end{aligned}
$$

By Theorem 1.1, the differential polynomial $g(z)=d_{2} f^{\prime \prime}+d_{1} f^{\prime}+d_{0} f$ satisfies $\bar{\lambda}(g-\varphi)=+\infty$.

Acknowledgement. The authors would like to thank the referees for their helpful comments and suggestions to improve our paper.

\section{References}

[1] Chen, Z. X., Zeros of meromorphic solutions of higher order linear differential equations, Analysis (Oxford) 14 (1994), 425-438.

[2] Chen, Z. X., The fixed points and hyper order of solutions of second order complex differential equations (in Chinese), Acta Math. Sci. Ser. A Chin. Ed. 20(3) (2000), 425-432.

[3] Chen, Z. X., Shon, K. H., On the growth and fixed points of solutions of second order differential equations with meromorphic coefficients, Acta Math. Sin. (Engl. Ser.) 21(4) (2005), 753-764.

[4] Gundersen, G. G., On the question of whether $f^{\prime \prime}+e^{-z} f^{\prime}+Q(z) f=0$ can admit a solution $f \not \equiv 0$ of finite order, Proc. Roy. Soc. Edinburg Sect. A 102 (1986), 9-17.

[5] Gundersen, G. G., Finite order solutions of second order linear differential equations, Trans. Amer. Math. Soc. 305 (1988), 415-429.

[6] Gundersen, G. G., Estimates for the logarithmic derivative of a meromorphic function, plus similar estimates, J. London Math. Soc. (2) 37 (1988), 88-104.

[7] Hayman, W. K., Meromorphic Functions, Clarendon Press, Oxford, 1964.

[8] Hayman, W. K., The local growth of power series: a survey of the Wiman-Valiron method, Canad. Math. Bull. 17 (1974), 317-358.

[9] Kinnunen, L., Linear differential equations with solutions of finite iterated order, Southeast Asian Bull. Math. 22(4) (1998), 385-405.

[10] Laine, I., Nevanlinna Theory and Complex Differential Equations, Walter de Gruyter, Berlin-New York, 1993.

[11] Laine, I., Rieppo, J., Differential polynomials generated by linear differential equations, Complex Var. Theory Appl. 49(12) (2004), 897-911 
[12] Liu, M. S., Zhang, X. M., Fixed points of meromorphic solutions of higher order Linear differential equations, Ann. Acad. Sci. Fenn. Math. 31 (2006), 191-211.

[13] Nevanlinna, R., Eindeutige Analytische Funktionen (in German), Zweite Auflage. Reprint. Grundlehren Math. Wiss. 46, Springer-Verlag, Berlin-New York, 1974.

[14] Valiron, G., Lectures on the General Theory of Integral Functions, Chelsea, New York, 1949.

[15] Wang, J. and Yi, H. X., Fixed points and hyper order of differential polynomials generated by solutions of differential equation, Complex Var. Theory Appl. 48(1) (2003), 83-94.

[16] Yang, C. C., Yi, H. X., Uniqueness theory of meromorphic functions, Math. Appl. 557, Kluwer Acad. Publ. Group, Dordrecht, 2003.

[17] Zhang, Q. T., Yang, C. C., The Fixed Points and Resolution Theory of Meromorphic Functions (in Chinese), Beijing Univ. Press, Beijing, 1988.

BENHARRAT BELAÏDI

DEPARTMENT OF MATHEMATICS

LABORATORY OF PURE

AND APPliEd MATHEMATiCS

UNIVERSITY OF MOSTAGANEM

B.P. 227 Mostaganem, Algeria

B.P. 227 Mostaganem, AlgEria
E-MAIL: EL.FARISSI.ABDALlAH@CARAMAIL.COM
AbDallah El FARISSI

DEPARTMENT OF MATHEMATICS

AND APPlied MATHEMATICS

UNIVERSITY OF MOSTAGANEM

B.P. 227 Mostaganem, Algeria
FARISSi.ABDALLAH@CARAMAIL.COM
LABoratory of Pure

E-MAIL: BELAIDI@UNIV-MOSTA.DZ

E-MAIL: BELAIDIBENHARRAT@YAHOO.FR 